\title{
Leigh Syndrome Due to mtDNA Pathogenic Variants
}

Journal of Inborn Errors

of Metabolism \& Screening

2019, Volume 7: e20180003

DOI: 10.1590/2326-4594-JIEMS-2018-0003

\section{Cristina Pereira ${ }^{1 *}$, Carolina Fischinger de Souza ${ }^{2 *}$, Leonardo Vedolin $^{3,6}$, Filippo Vairo ${ }^{3,5}$, Cláudia Lorea ${ }^{3,7}$, Cláudia Sobreira ${ }^{4}$, Célia Nogueira' ${ }^{1}$ (i) and Laura Vilarinho'}

\begin{abstract}
Leigh syndrome is a devastating neurodegenerative disease, typically manifesting in infancy or early childhood. Hallmarks of the disease are symmetrical lesions in the basal ganglia or brain stem on MRI, and a clinical course with rapid deterioration of cognitive and motor functions. It is genetically heterogeneous, causative mutations have been disclosed in mitochondrial DNA and nuclear genes involved in the process of energy production in the mitochondria. We investigated the whole mitochondrial DNA in three Brazilian patients with LS, based on their clinical and biochemical data, with the aim to identify the disease-causing mutations. In two of the patients, with complex I deficiency, a novel heteroplasmic variant m.4142G>T (p.R279L) in MT-ND1 and a recurrent homoplasmic mutation m.10197G>A (p.A47T) in MT-ND3 were identified. In the remaining patient, with complex IV deficiency, a de novo heteroplasmic variant in MT-CO1 m.6547T>C (p.L215P) was found. The molecular investigation in mitochondrial diseases have shifted their focus from mitochondrial DNA to nuclear DNA, however, mtDNA protein-coding genes are one of the important genetic causes of mitochondrial disorders for Leigh syndrome. This study expands the molecular and clinical spectrum associated with this disease.
\end{abstract}

\section{Keywords}

leigh syndrome, mitochondrial genome, complex I, complex IV, MT-ND3, MT-CO1

\section{Introduction}

Leigh syndrome (LS) or subacute necrotising encephalopathy (OMIM \#256000) is a progressive neurodegenerative disorder, typically manifesting in infancy or early childhood. Since its first description by Denis Archibald Leigh in 1951, ${ }^{[1]}$ it has evolved from a postmortem diagnosis, strictly defined by histopathological observations, to a clinical entity characterized by neurodegenerative symptoms, typical characteristic magnetic resonance imaging (MRI) lesions and heterogenic genetic causes of deficient mitochondrial energy metabolism. Hallmarks of the disease are symmetrical lesions in the basal ganglia or brain stem on MRI, and a clinical course with rapid deterioration of cognitive and motor functions. ${ }^{[1,2]}$

Patients with LS have a wide spectrum of clinical presentations including hypotonia, ataxia, dystonia, epilepsy, respiratory abnormalities, nystagmus, optic atrophy and ophthalmoplegia. Failure to thrive or developmental retardation is often an early but unspecific sign of the disease. Stepwise deterioration with some recovery of skills between episodes of regression is common. The severe neurological symptoms are caused by a range of different biochemical and molecular defects interfering with cellular energy production in affected brain regions. ${ }^{[3]}$ Non-specific biochemical

\footnotetext{
${ }^{1}$ Departamento de Genética Humana, Instituto Nacional de Saúde Doutor Ricardo Jorge, Porto, Portugal

2 Departamento de Genética Médica, Hospital de Clinicas de Porto Alegre, Porto Alegre, RS, Brazil

${ }^{3}$ Department of Clinical Genomics, Mayo Clinic, Rochester, MN, USA

${ }^{4}$ Departamento de Neurociências, Universidade de São Paulo, Ribeirão Preto, SP, Brazil

${ }^{5}$ Center for Individualized Medicine, Mayo Clinic, Rochester, MN, USA

${ }^{6}$ DASA, Barueri, SP, Brazil

${ }^{7}$ Hospital Escola, Universidade Federal de Pelotas, Pelotas, RS, Brazil
}

* These authors contributed equally to this work

Received November 14, 2018, and in revised form April 18, 2019. Accepted for publication May 15, 2019.

\section{Corresponding Author:}

Carolina Fischinger de Souza, Medical Genetics Department, Hospital de Clinicas de Porto Alegre, Brazil, R. Ramiro Barcelos, 2350 - Santa Cecilia, Porto Alegre - RS, 90035-903, Brazil, +55 51 3359-8000.

Email: cfsouza@hcpa.edu.br 
findings include increased lactate in cerebral spinal fluid and blood and increased Krebs cycle intermediates in urine, pointing to a deficit in energy metabolism. Multiple genetic causes have been described in LS comprising all aspects of energy generation including mutations in mitochondrial DNA (mtDNA) and in nuclear genes encoding the proteins involved in the structural components of the respiratory chain complexes, the electron carrier of coenzyme Q10, assembly proteins of the various complexes and super complexes, protein transporters, and modifiers of mtRNAs. Although most patients with LS have a mutation in nuclear DNA, about $25 \%$ have a mutation in mtDNA. ${ }^{[4]}$

Complex I (CI) deficiency is assumed the most common cause of respiratory chain deficiency in humans, and is also the most common cause of LS associated with numerous pathogenic mutations in either mtDNA or in nuclear-encoded complex I structural subunits or assembly chaperone proteins. Recent studies indicate that mutations in mtDNA can be causative in up to $30 \%$ of complex I-deficient individuals, and recurrent mutations in a small number of the mitochondrially encoded complex I genes (MT-ND) account for a substantial proportion of these cases contributing to the broad clinical spectrum of disease phenotypes including LS. ${ }^{[5]} \mathrm{A}$ mitochondrial hotspot gene is MT-ATP6, which account with the most frequently described mutation associated with LS, the m.8993T>G/C. ${ }^{[6]}$ In contrast with MT-ND mutations just a handful of mutations have been reported in mitochondrially encoded complex IV genes (MT-CO), however mutations in the SURF1 are one of the most common nuclear mutations consistently associated with LS and cytochrome c oxidase (COX) deficiency. ${ }^{[7]}$

Mitochondrial respiratory chain (MRC) enzyme assay and gene analysis are crucial for a precise etiological diagnosis of the disease. The identification of the causal mutation is clinically important, as it provides a diagnosis for the affected family. Here, we describe three Brazilian patients with LS in whom we probably identified the disease-causing variants.

\section{Patients}

Patient 1 - A 4-year-old boy referred to genetic evaluation due to basal ganglia lesions and suspicion of genetic or infectious disorders. He is the first child, from a non-consanguineous couple, who was born from a caesarian section without intercurrence and without history of genetic disorders in the family. The couple has another healthy daughter. The language was delayed and started with 2,5 years. At 4 years he was submitted to an adenoid surgery and after the procedure he started with ataxia and walk incoordination. After neurological evaluation the MRI showed T2 hyper signal in putamen, pallidal globus, tallamus and diffusion destriction in basal ganglia. The metabolic evaluation was normal. Biochemical assays of MRC activities in muscle tissue homogenate showed an isolated complex I deficiency. The clinical evaluation revealed spastic deambulation with cerebellar signs, diffuse hypereflexia, clonus, dysarthria, dystonia, very mild cognitive impairment. The patient is stable, using riboflavin, vitamin C and Coenzyme Q10.
Patient 2 - A 17-month-old boy with history of poor weight gain, seizures and recurrent pneumonia was referred to us by a local hospital for genetics evaluation. He was born at full term by vaginal delivery of healthy, non-consanguineous parents. He was the couples' first born; his father already had a 10 -year-old boy with hemophilia from a previous relationship. Around 3 to 4 months of age he was hypotonic, he did not achieve milestones as expected for his age, he also presented poor weight gain. At 11 months he started having seizures and he was treated with phenobarbital with initial good control. He became underweight and at 12 months of age a percutaneous endoscopic gastrostomy was performed. On physical examination, at age 17 months, hyporeflexia and severe hypotonia was observed. Plasma lactate was $12.1 \mathrm{mmol} / \mathrm{L}$ (normal range $<2,2 \mathrm{mmol} / \mathrm{L}$ ). Enzymatic analysis of the muscle demonstrated an isolated complex I deficiency. Basal ganglia lesions were identified on brain-CT (Fig. 1), but unfortunately a MRI could not be performed. His clinical symptoms continued to progress and he was admitted to the hospital in status epilepticus and died 20 days later, with 3 years and 2 months of age.

Patient 3 - A 23-month-old-boy was referred to medical genetic consultation with a complaint of severe hypotonia. He was the second liveborn child of a healthy nonconsanguineous couple, with no particular familial history. On clinical examination, both parents and the older sister are asymptomatic. He sat at 8 months of age, he spoke first words at 18 months of age and he stand after 23 months. On general clinical examination, he had no dysmorphic features, without palpebral ptosis. He was hypotonic with undescended testis. During investigation, his auditory exams demonstrated a retrocochlear alteration. His first MRI showed basal ganglia hyperintensity on T2-weighted images (Fig. 2A). The plasma lactate was $7.0 \mathrm{mmol} / \mathrm{L}$ (normal range: $<2.2 \mathrm{mmol} / \mathrm{L}$ ) and in amino acid profile the alanine was increased. After 3 years, he presented focal neurological deficit in the right side. At that time, the MRI showed cortical changes, especially in left hemisphere, incongruent to a vascular territory with focal hyperintensities in basal ganglia besides an inverted peak of lactate in proton spectroscopy (Fig. 2B). Muscle biopsy was performed to biochemical analysis MRC and it showed a significant
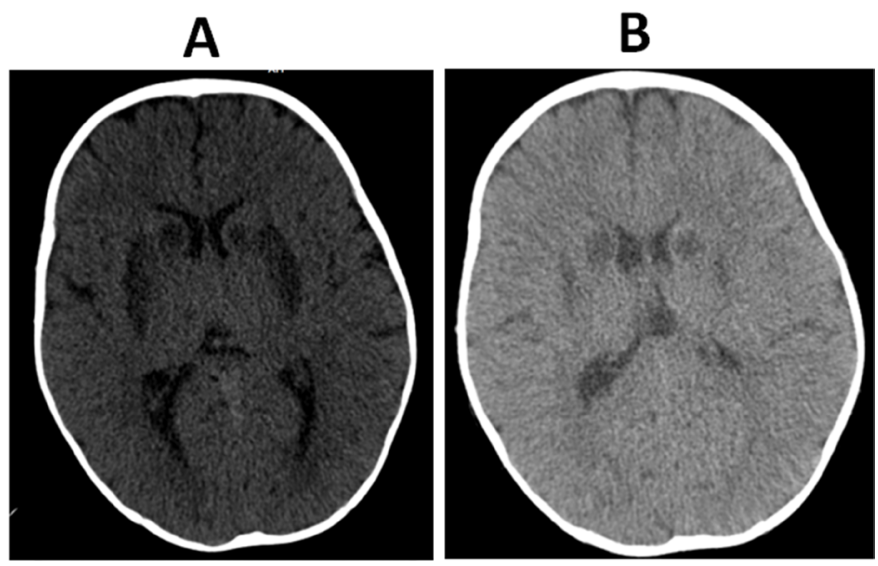

Figure 1. Axial nonenhanced computed tomography (CT) shows (A) extensive and (B) moderate vascular lesions affecting the basal ganglia. 

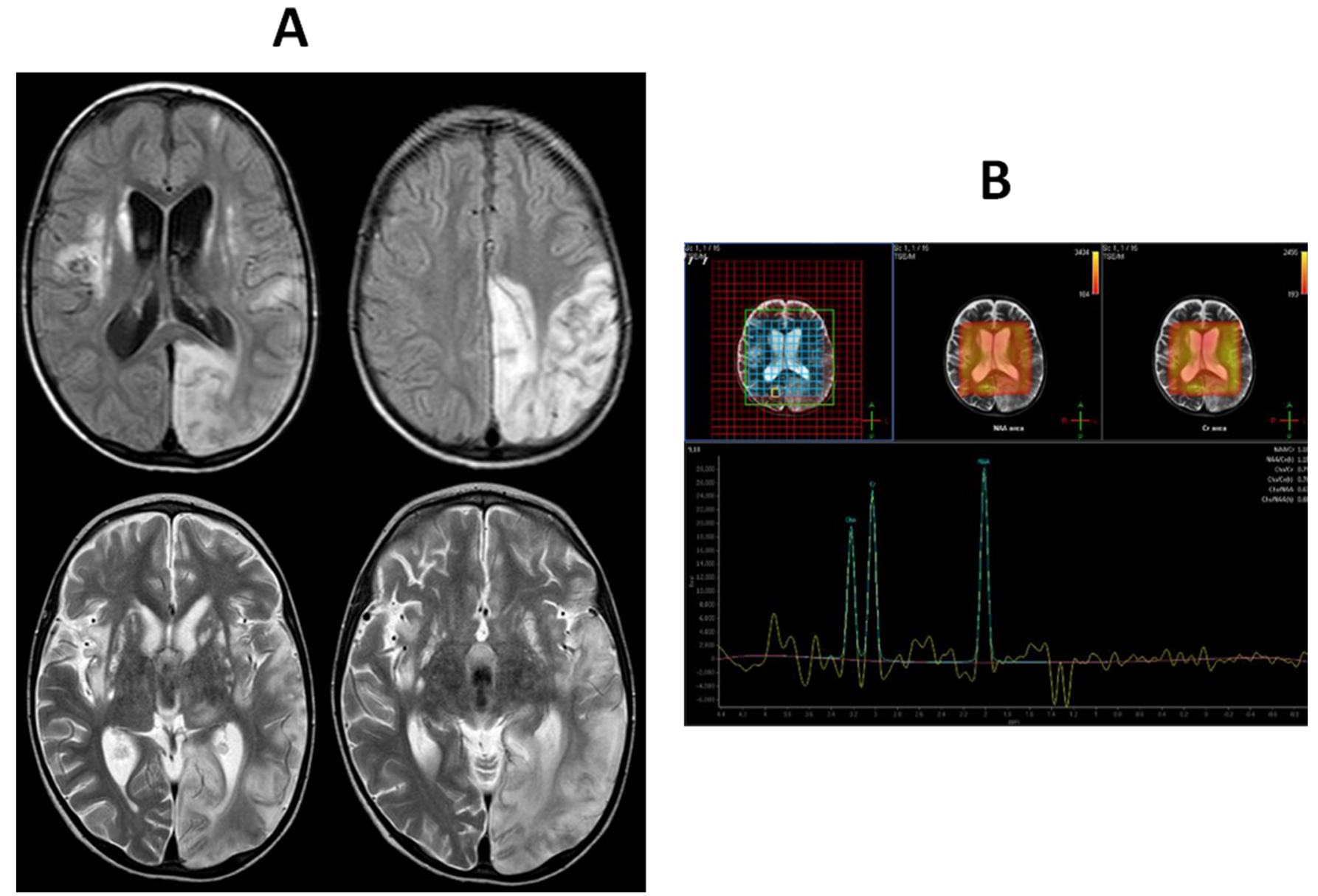

Figure 2. (A) Flair and T2-weighted MRI images showing cortical changes, especially in left hemisphere, incongruent to a vascular territory, with focal hyperintensities in basal ganglia; (B) Proton spectroscopy showing inverted peak of lactate at 1.3 ppm.

reduction of the complex IV (CIV) and mild decrease of complex II+III activities. One year later, he developed Wolf-ParkinsonWhite syndrome and nowadays he is fed by gastrostomy. At physical examination, he does not walk and he presents ataxia, incoordination, axial hypotonia and appendicular hypertonia.

\section{Methods}

Total DNA was extracted from muscle biopsy homogenates using standard procedures. ${ }^{[8]}$ The entire mtDNA was amplified using 46 overlapping primers from Applied Biosystems (Variant $\mathrm{SEQr}^{\mathrm{TM}}$ resequencing System) for patients 1 and 2, and MT-CO genes were amplified with the corresponding primers from the same kit, for patient 3. Sanger sequencing of PCR products were carried out with the BigDye v3.1 terminator cycle sequencing methodology on an ABI PRISM ${ }^{\mathrm{TM}}$ 3130XL Genetic Analyser (Applied Biosystems). ${ }^{\left[{ }^{[9]}\right.}$ The sequences obtained were edited and aligned with the Cambridge Reference Sequence and the consensus was made using the SeqScape software v2.5 (Applied Biosystems). All the variations along with their position were noted and searched in the human mitochondrial genome databases such as Mitomap (http: //www.miomap.org), mtDB (http: //www.genpat.uu.se/mtDB) and HmtDB (htpp: //www. hmtdb.uniba.it:8080/hmdb) for their significance. The mutations were submitted to MToolBox, APOGEE, Mitoclass.1 and HmtVar for a prediction of pathogenicity. These recent tools were developed for mtDNA using machine learning based approaches. ${ }^{[10]}$ The HmtVar explore human mitochondrial variability data and their pathological correlations. The phylogenetic analysis and the mammalian conservation index (MCI) were also evaluated.

\section{Results}

\section{Patient 1}

A novel missense variant $\mathrm{m} .4142 \mathrm{G}>\mathrm{T}$ (p.R279L) was detected in $M T-N D 1$ in the muscle of this patient, with a mutant load of near 90\% (Fig. 3A). This variant changes an arginine residue into a leucine (p.Arg279Leu). Patient's mother was unaffected and did not carry measurable mutant mtDNA in blood (Fig. 3B). The variant was also absent in the patient's sibling (Fig. 3C). This heteroplasmic variant was not present in the human mitochondrial genome databases and was not detected in about $500 \mathrm{mtDNA}$ genomes previously sequenced by our group. On phylogenetic analysis, the Arg279 amino acid is completely conserved across different 39 species corresponding to a conservation index of $100 \%$. This 
evolutionary conserved residue substitution is located in an extra membrane loop of the ND1 subunit of MRC complex I (Fig. 4). The variant was predicted to be damaging by in silico analysis (MToolBox, APOGEE, Mitoclass.1 and HmtVar).

\section{Patient 2}

The whole mitochondrial DNA sequencing from skeletal muscle of the patient revealed, in apparent homoplasmy, the already reported $\mathrm{m} .10197 \mathrm{G}>\mathrm{A}$ (p.A47T) in MT-ND3. ${ }^{[1]}$ This mutation modifies a hydrophobic alanine residue into a hydrophilic threonine in a highly conserved domain of MT-ND3 subunit. Any family members were investigated.

\section{Patient 3}

Sequence analysis of MT-CO genes from the patient muscle showed a de novo missense m.6547T $>\mathrm{C}$ (p.L215P) in the MTCO1, with a heteroplasmy level of approximately $50 \%$ (Fig. 5A). This variant was not detected in his mother (Fig. 5B). The variant results in the substitution of a highly conserved leucine to proline at codon 215 , this residue have a mammalian conservation index (MCI) of $100 \%$. The p.L215P is located in an extramembrane loop of the CO1 subunit of complex IV of MRC, in a region highly conserved through phylogenesis (Fig. 6). The variant was predicted to be damaging by bioinformatics analysis (MToolBox, APOGEE, Mitoclass.1 and HmtVar).

\section{A}

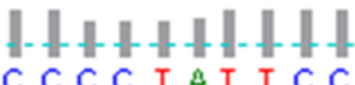

m.4142G $>T$

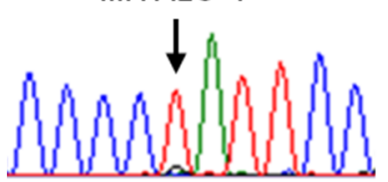

B

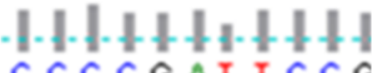

$C C C C G A T T C C G$

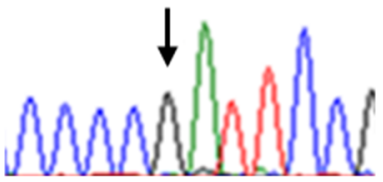

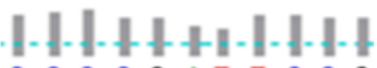

C C C G AT T C C G

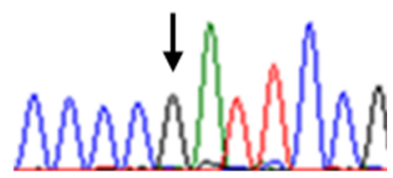

Figure 3. (A) Electropherogram showing the $m .4142 \mathrm{G}>\mathrm{T}$ in patient 1 ; (B) Electropherogram of his mother; (C) Electropherogram of his sibling.

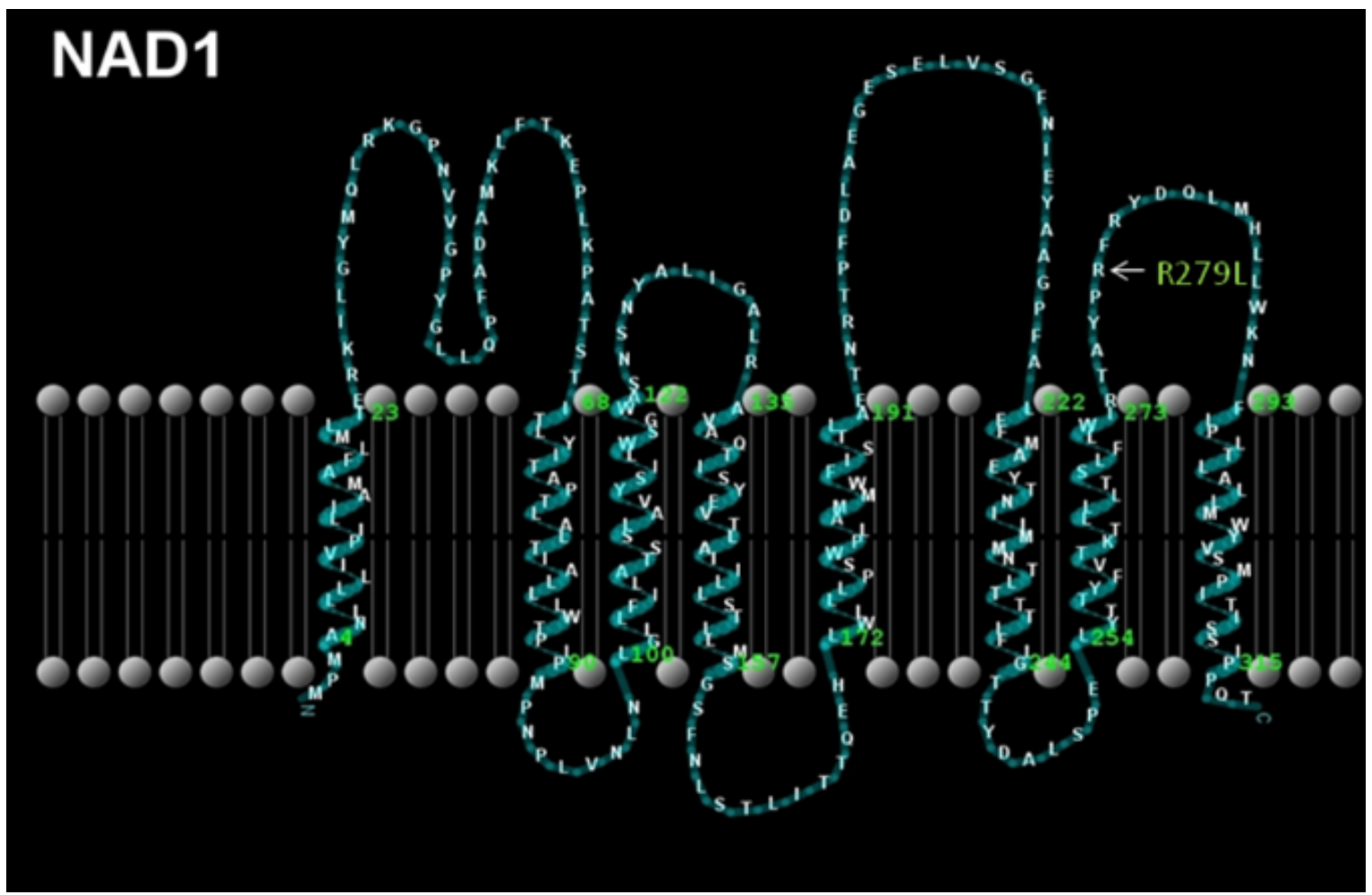

Figure 4. Localization of the $m .4142 \mathrm{G}>\mathrm{T}$ (p.R279L) on the MT-ND1. The biochemical activity of the ND1 subunit of complex I is deficient in patient 1. 

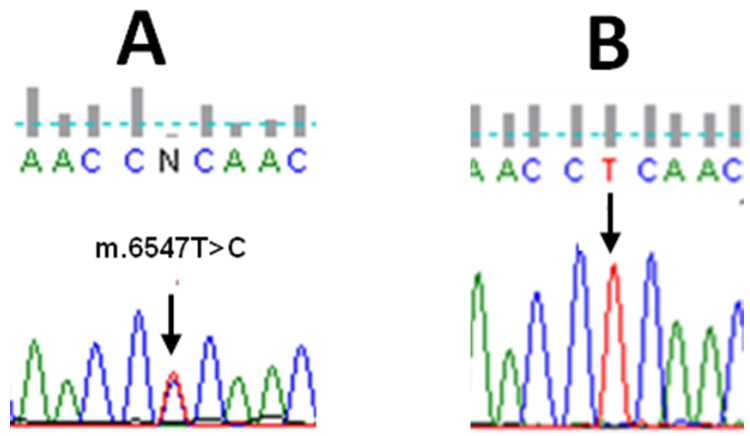

Figure 5. (A) Electropherogram showing the m.6547T>C in patient 3; (B) Electropherogram of his mother.

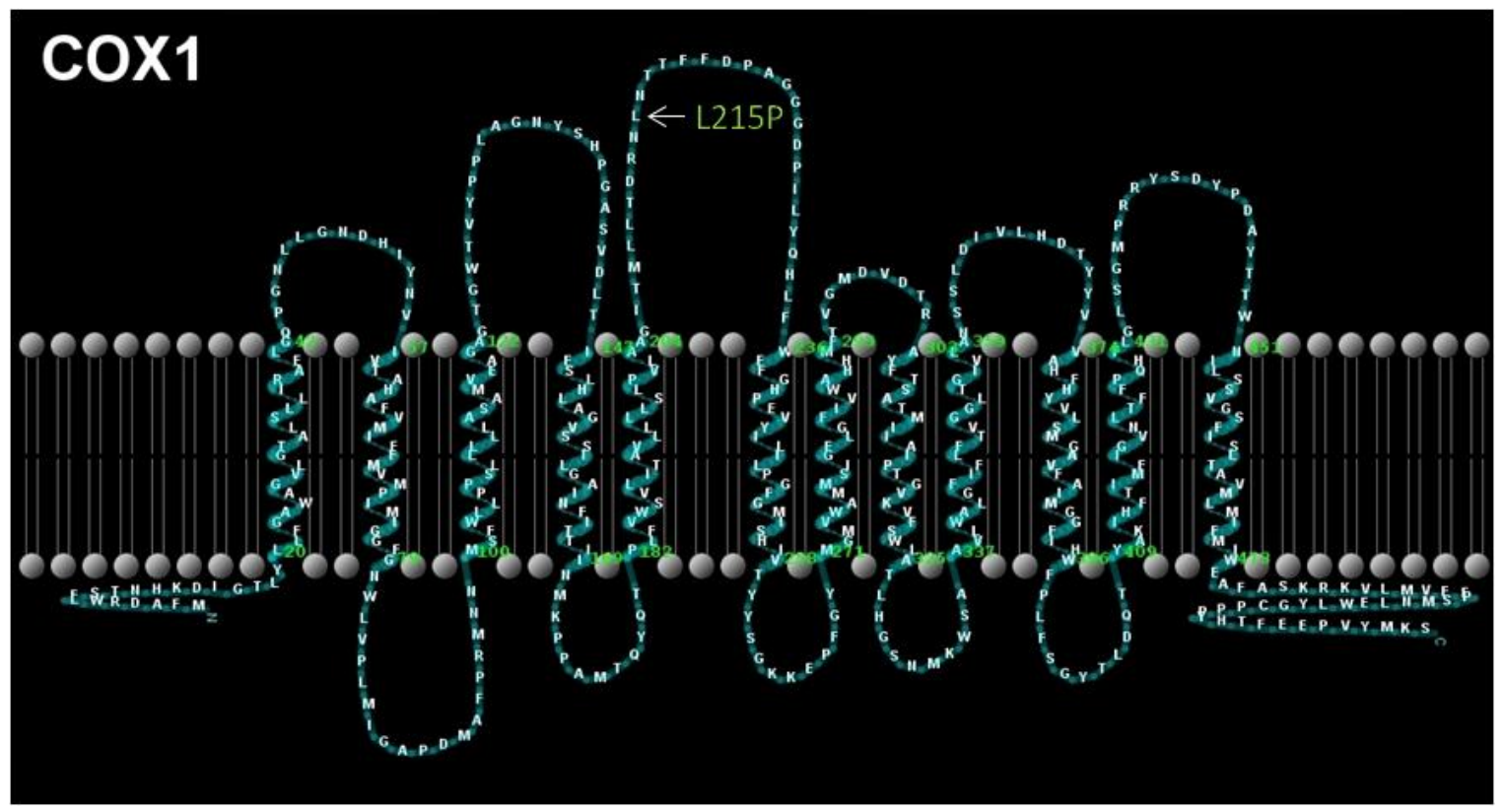

Figure 6. Localization of the m.6547T>C (p.L251P) on the MT-CO1. The biochemical activity of the CO1 subunit of complex IV is deficient in patient 3.

\section{Discussion}

LS mutations affect genes of either the mitochondrial or nuclear genome. ${ }^{[7]}$ MtDNA mutations are a frequent cause of LS (Table 1) and unexpectedly, nuclear and mtDNA mutations cause similar LS disease phenotypes associated with early onset and poor prognosis. ${ }^{[3]}$

Mutations in each of the seven MT-ND encoded subunits have been reported representing 20 to $30 \%$ of the causes of isolated complex I deficiency. Several recurrent de novo complex I mtDNA mutations that cause Leigh-like disease have been described, but novel mutations are rarely reported. ${ }^{[39]}$

Here, we report three Brazilian boys with LS in whom we identified mutations in MT-ND1, MT-ND3 and MT-CO1: the novel heteroplasmic variant $\mathrm{m} .4142 \mathrm{G}>\mathrm{T}$ in patient 1 , the previously reported $\mathrm{m} .10197 \mathrm{G}>\mathrm{A}$ mutation in patient 2 and a de novo variant $\mathrm{m} .6547 \mathrm{~T}>\mathrm{C}$ in patient 3 , respectively.

The novel $\mathrm{m} .4142 \mathrm{G}>\mathrm{T}$ variant changes a basic polar arginine into a nonpolar leucine at codon 279 (p.A279L). At this residue another mutation has been reported as pathogenic, the m.4142G>A (p.R279Q), in a ten-year-old patient who presented with developmental delay, seizure and hypotonia. ${ }^{[40]}$ All known MT-ND1 pathogenic mutations occur in intra mitochondrial loops, such binding sites are usually highly conserved in evolution as indeed are the amino acids surrounding the affected residue. ${ }^{[15]}$ The novel $\mathrm{m} .4142 \mathrm{G}>\mathrm{T}$ variant is localized in such a region which probably have functional consequences. Mutations in mtDNA can cause LS when sufficiently abundant or severe enough to impair oxidative phosphorylation, which was verified in patient 1 since the mutant load in muscle is 
Table 1. Mutations in mitochondrial genes causing maternally inherited Leigh syndrome

\begin{tabular}{|c|c|c|c|}
\hline Gene & Mutation & Other Phenotypes & References \\
\hline MTTV & $\mathrm{m} .1644 \mathrm{G}>\mathrm{T}$ & LS; MELAS & [12] \\
\hline MTTL & $\mathrm{m} .3243 \mathrm{~A}>\mathrm{G}$ & MELAS; LS & [13] \\
\hline MTND1 & $\mathrm{m} .3697 \mathrm{G}>\mathrm{A}$ & MELAS; LS & [15] \\
\hline MTND2 & $\mathrm{m} .4681 \mathrm{~T}>\mathrm{C}$ & LS & [16] \\
\hline MTTK & $\mathrm{m} .8363 \mathrm{G}>\mathrm{A}$ & LS; MERRF; DEAF & [19] \\
\hline MTATP6 & $\mathrm{m} .8993 \mathrm{~T}>\mathrm{G}$ & LS; NARP & [20] \\
\hline MTATP6 & $m .8993 T>C$ & LS; NARP & [21] \\
\hline MTATP6 & $\mathrm{m} .9176 \mathrm{~T}>\mathrm{G}$ & LS & [22] \\
\hline MTCO3 & m.9537ins C & LS-like & [25] \\
\hline МТСО3 & $\mathrm{m} .9547 \mathrm{G}>\mathrm{A}$ & LS & [25] \\
\hline MTND3 & m.10158T>C & LS & [26] \\
\hline MTND3 & $\mathrm{m} \cdot 10191 \mathrm{~T}>\mathrm{C}$ & LS; LS-like; ESOC & [27] \\
\hline MTND3 & m.10197G >A & LS; LDYT; MELAS & [15] \\
\hline MTND4 & $\mathrm{m} .11777 \mathrm{C}>\mathrm{A}$ & LS & [28] \\
\hline MTND4 & m.11984T>C & LS & [29] \\
\hline MTND5 & m.12706T>C & LS & [30] \\
\hline MTND5 & m.13045A>C & MELAS; LHON; LS & [31] \\
\hline MTND6 & m.14600G>A & LS; Optic atrophy & [38] \\
\hline
\end{tabular}

DEAF - Deafness, Sensorineural Hearing Loss; ESOC - Epilepsy, Strokes, Optic atrophy, and Cognitive decline; FBSN - Familial Bilateral Striatal Necrosis; LDYT - Leber Hereditary Optic Neuropathy and Dystonia; LHON - Leber Hereditary Optic Neuropathy; LS - Leigh Syndrome; MELAS - Mitochondrial Encephalomyopathy, Lactic Acidosis and Stroke-Like Episodes; MERRF - Myoclonic Epilepsy and Ragged Red Fiber; NARP - Neurogenic Muscle Weakness, Ataxia, and Retinitis Pigmentosa.

approximately $90 \%$ and severely impaired complex I activity. Because single fiber studies and cybrid data were not available to assess the pathogenicity of this variant, we classified it as probably deleterious as it met the following criteria: (1) is a missense variant occurring at an evolutionarily highly conserved amino acid (2) the change is novel and has not been observed in normal controls (3) is in heteroplasmic state and has a high mutant load in the affected tissue (4) is in MT-ND1 and the patient has a deficiency of complex I (5) occurs at a structurally and functionally conserved region of the protein (6) is predicted to be damaging by in silico algorithms (MToolBox, APOGEE, Mitoclass.1 and HmtVar) (7) patient clinical presentation.
The recurrent m.10197G $>$ A mutation in $M T-N D 3$ has been already described in French and Korean LS patients, ${ }^{[1,41]}$ associated with complex I deficiency and a severe clinical phenotype, as we report for patient 2 , in a near homoplasmic state.

In patient 3 , with an isolated deficiency of $\mathrm{CIV}$, we identified a de novo heteroplasmic variant m.6547T $>C$ (p.L251P) in MTCO1. In contrast with MT-ND mutation, only a small number of mutations have been reported as pathogenic in MT-CO genes. Mutations in this complex IV subunit are usually sporadic, very rare and have been associated with a spectrum of conditions including sideroblastic anemia, myopathy with myoglobinuria 
and, stroke-like episodes, leading to severe, drug-refractory epilepsy. ${ }^{[42]}$ The m.6547T $>\mathrm{C}$ variant has already been reported in a patient with Myoclonus Epilepsy with Ragged-Red Fibers (MERRF) in whom the m.8344A $>\mathrm{G}$ was detected. Interestingly in this article it was suggested that not one but the type and total number of mutations is an indispensable factor for the disease. ${ }^{[43]}$ Unfortunately it was not possible to sequence all the mitochondrial genome in patient 3 . Even though it has been reported as a variant, the present study strongly argue for the role of $\mathrm{m} .6547 \mathrm{~T}>\mathrm{C}$ as a causative mutation. The m.6547T $>\mathrm{C}$ was detected with a mutant load of about $50 \%$ in the muscle of this patient who has an isolated deficiency of CIV and is localized in a conserved region of MT-COI. Although the average level of the mutation and the amino acid substitution are not significant, the m.6547 $>\mathrm{C}$ may play a role on the phenotype presented since it is related with the deficiency found on patient 3 and it has been referred that mutations in the mitochondrial subunits of CIV do not need a high mutant load to cause impair oxidative phosphorylation. COX I is the first subunit to be inserted in the inner mitochondrial membrane during COX assembly, followed by incorporation of COX III and COX II. It was indicated that altered COX I species are unable to drive the incorporation of the other subunits into the nascent COX holoenzyme, thus inducing their degradation. ${ }^{7}$ Further studies can be necessary to confirm the solely pathogenicity of the m.6547T $>C$ or if it has a role in the penetrance of other genetic defects on mitochondrial or nuclear genes.

\section{Conclusion}

Mutations of mtDNA are an important cause of LS and these additional cases reinforce this fact and expands the molecular and clinical spectrum. MT-ND genes are relevant mutation hot spots in pediatric patients with complex I deficiency accompanied by variable severity of mitochondrial encephalopathy as described in two of these patients. In the MT-CO1 gene deleterious mutations have been rarely identified, we probably found such a variant in the third patient which is in concordance with the MRC complex IV deficiency. Identifying a new mutation as pathogenic in a protein-coding gene on mtDNA is difficult given the large number of polymorphisms that can occur but its identification is clinically important as it provides a diagnosis for the affected family. Therefore, we suggest that mtDNA analysis by Next Generation Sequencing (NGS) should be included in the diagnosis of LS patients as a first approach, since it is more timely and cost effective. In the negative cases the nuclear genes should be screened by NGS (clinical exome/whole exome sequencing).

\section{Ethics Approval and Consent to Participate}

All procedures followed were in accordance with the ethical standards of the responsible committee on human experimentation (institutional and national) and with the Helsinki Declaration of 1975 , as revised in 2000 . Informed consent was obtained from all patients for being included in this study.

\section{Declaration of Conflicting Interests}

The author(s) declared no potential conflicts of interest with respect to the research, authorship, and/or publication of this article.

\section{References}

1. Leigh D. Subacute necrotizing encephalomyelopathy in an infant. J Neurol Neurosurg Psychiatry. 1951;14(3):216-221. doi:10.1136/jnnp.14.3.216

2. Baertling F, Schaper J, Mayatepek E, Distelmaier F. Teaching NeuroImages: rapidly progressive leukoencephalopathy in mitochondrial complex I deficiency. Neurology. 2013;81(2):e10-e11. doi:10.1212/WNL.0b013e31829a339b

3. Naess K, Freyer C, Bruhn H, et al. MtDNA mutations are a common cause of severe disease phenotypes in children with Leigh syndrome. Biochim Biophys Acta. 2009;1787(5):484490. doi:10.1016/j.bbabio.2008.11.014

4. Ruhoy IS, Saneto RP. The genetics of Leigh Syndrome and its implications for clinical practice and risk management. Appl Clin Genet. 2014;13(7):221-234. doi:10.2147/TACG.S46176

5. Nesbitt V, Morrison PJ, Crushell E, et al. The clinical spectrum of the m.10191T $>$ C mutation in complex I-deficient Leigh syndrome. Dev Med Child Neurol. 2012;54(6):500-506. doi:10.1111/j.1469-8749.2012.04224.x

6. Finsterer J. Leigh and Leigh-like syndrome in children and adults. Pediatr Neurol. 2008;39(4):223-235. doi:10.1016/j. pediatrneurol.2008.07.013

7. Valente L, Piga D, Lamantea E, et al. Identification of novel mutations in five patients with mitochondrial encephalomyopathy. Biochim Biophys Acta. 2009;1787(5):491501. doi:10.1016/j.bbabio.2008.10.001

8. Bugiani $\mathrm{M}$, Invernizzi F, Alberio $\mathrm{S}$, et al. Clinical and molecular findings in children with complex I deficiency. Biochim Biophys Acta. 2004;1659(2-3):136-147. doi:10.1016/j. bbabio.2004.09.006

9. Choi BO, Hwang JH, Cho EM, et al. Mutational analysis of whole mitochondrial DNA in patients with MELAS and MERRF diseases. Exp Mol Med. 2010;42(6):446-455. doi:10.3858/emm.2010.42.6.046

10. Bris C, Goudenege D, Desquiret-Dumas V, et al. Bioinformatics Tools and Databases to Assess the Pathogenicity of Mitochondrial DNA Variants in the Field 
of Next Generation Sequencing. Front Genet. 2018;9:632 doi:10.3389/fgene.2018.00632

11. Sarzi E, Brown M, Lebon S, et al. A novel recurrent mitochondrial DNA mutation in ND3 gene is associated with isolated complex I deficiency causing Leigh syndrome and dystonia. Am J Med Genet. 2007;143(A):33-41. doi:10.1002/ ajmg.a.31565

12. Goldenberg PC, Steiner RD, Merkens LS, et al. Remarkable improvement in adult Leigh syndrome with partial cytochrome c oxidase deficiency. Neurology. 2003;60(5):865868. doi:10.1212/01.WNL.0000049460.72439.7F

13. Vilarinho L, Santorelli FM, Coelho I, et al. The mitochondrial DNA A3243G mutation in Portugal: clinical and molecular studies in 5 families. J Neurol Sci. 1999;163(2):168-174. doi:10.1016/S0022-510X(99)00030-1

14. Funalot B, Reynier P, Vighetto A, et al. Leigh-like encephalopathy complicating Leber's hereditary optic neuropathy. Ann Neurol. 2002;52(3):374-377. doi:10.1002/ ana.10299

15. Kirby DM, McFarland R, Ohtake A, et al. Mutations of the mitochondrial ND1 gene as a cause of MELAS. J Med Genet. 2004;41(10):784-789. doi:10.1136/jmg.2004.020537

16. Ugalde C, Hinttala R, Timal S, et al. Mutated ND2 impairs mitochondrial complex I assembly and leads to Leigh syndrome. Mol Genet Metab. 2007;90(1):10-14. doi:10.1016/j. ymgme.2006.08.003

17. Santorelli FM, Tanji K, Sano M, et al. Maternally inherited encephalopathy associated with a single-base insertion in the mitochondrial tRNATrp gene. Ann Neurol. 1997;42(2):256260. doi:10.1002/ana.410420220

18. Howell N, Kubacka I, Smith R, Frerman F, Parks JK, Parker WD. Association of the mitochondrial 8344 MERRF mutation with maternally inherited spinocerebellar degeneration and Leigh disease. Neurology. 1996;46(1):219-222. doi:10.1212/ WNL.46.1.219

19. Shtilbans A, Shanske S, Goodman S, et al. G8363A mutation in the mitochondrial DNA transfer ribonucleic acid Lys gene: another cause of Leigh syndrome. J Child Neurol. 2000;15(11):759-761. doi:10.1177/088307380001501109

20. Holt IJ, Harding AE, Petty RK, et al. A new mitochondrial disease associated with mitochondrial DNA heteroplasmy. Am J Hum Genet. 1990;46:428-433.

21. de Vries DD, Van Engelen BG, Gabreels FJ, Ruitenbeek $\mathrm{W}$, van Oost BA. A second missense mutation in the mitochondrial ATPase 6 gene in Leigh's syndrome. Ann Neurol. 1993;34(3):410-412. doi:10.1002/ana.410340319

22. Carrozzo R, Murray J, Santorelli FM, Capaldi RA. The T9176G mutation of human mtDNA gives a fully assembled but inactive ATP synthase when modeled in Escherichia coli. FEBS Lett. 2000;486(3):297-299. doi:10.1016/S00145793(00)02244-4
23. Thyagarajan D, Shanske S, Vazquez-Memije M, Devivo D, Dimauro S. A novel mitochondrial ATPase 6 point mutation in familial bilateral striatal necrosis. Ann Neurol. 1995;38(3):468-472. doi:10.1002/ana.410380321

24. Moslemi AR, Darin N, Tulinius M, Oldfors A, Holme E. Two new mutations in the MTATP6 gene associated with Leigh syndrome. Neuropediatrics. 2005;36(5):314-318. doi:10.1055/s-2005-872845

25. Tiranti V, Corona P, Greco M, et al. A novel frameshift mutation of the mtDNA COIII gene leads to impaired assembly of cytochrome c oxidase in a patient affected by Leigh-like syndrome. Hum Mol Genet. 2000;9(18):2733-2742. doi:10.1093/hmg/9.18.2733

26. Crimi M, Papadimitriou A, Galbiati S, et al. A new mitochondrial DNA mutation in ND3 gene causing severe Leigh Syndrome with early lethality. Pediatr Res. 2004;55(5):842-846. doi:10.1203/01. PDR.0000117844.73436.68

27. Taylor RW, Singh-Kler R, Hayes CM, Smith PE, Turnbull DM. Progressive mitochondrial disease resulting from a novel missense mutation in the mitochondrial DNA ND3 gene. Ann Neurol. 2001;50(1):104-107. doi:10.1002/ana.1084

28. Komaki $\mathrm{H}$, Akanuma J, Iwata $\mathrm{H}$, et al. A novel mtDNA C11777A mutation in Leigh syndrome. Mitochondrion. 2003;2(4):293-304. doi:10.1016/S1567-7249(03)00003-5

29. Vanniarajan A, Rajshekher GP, Joshi MB, Reddy AG, Singh L, Thangaraj K. Novel mitochondrial mutation in the ND4 gene associated with Leigh syndrome. Acta Neurol Scand. 2006;114(5):350-353. doi:10.1111/j.1600-0404.2006.00673.x

30. Taylor RW, Morris AA, Hutchinson M, Turnbull DM. Leigh disease associated with a novel mitochondrial DNA ND5 mutation. Eur J Hum Genet. 2002;10(2):141-144. doi:10.1038/ sj.ejhg. 5200773

31. Liolitsa D, Rahman S, Benton S, Carr LJ, Hanna MG. Is the mitochondrial complex I ND5 gene a hot-spot for MELAS causing mutations? Ann Neurol. 2003;53(1):128132. doi:10.1002/ana.10435

32. Crimi M, Galbiati S, Moroni I, et al. A missense mutation in the mitochondrial ND5 gene associated with a LeighMELAS overlap syndrome. Neurology. 2003;60(11):18571861. doi:10.1212/01.WNL.0000066048.72780.69

33. Blok MJ, Spruijt L, de Coo IF, Schoonderwoerd K, Hendrickx A, Smeets HJ. Mutations in the ND5 subunit of complex I of the mitochondrial DNA are a frequent cause of oxidative phosphorylation disease. J Med Genet. 2007;44(4):e74. doi:10.1136/jmg.2006.045716

34. Santorelli FM, Tanji K, Kulikova R, et al. Identification of a novel mutation in the mtDNA ND5 gene associated with MELAS. Biochem Biophys Res Commun. 1997;238(2):326328. doi:10.1006/bbrc.1997.7167 
35. Corona P, Antozzi C, Carrara F, et al. A novel mtDNA mutation in the ND5 subunit of complex I in two MELAS patients. Ann Neurol. 2001;49(1):106-110. doi:10.1002/15318249(200101)49:13.0.CO;2-T

36. Jun AS, Brown MD, Wallace DC. A mitochondrial DNA mutation at np 14459 of the ND6 gene associated with maternally inherited Leber's hereditary optic neuropathy and dystonia. Proc Natl Acad Sci USA. 1994;91(13):62066210. doi:10.1073/pnas.91.13.6206

37. Solano A, Roig M, Vives-Bauza C, et al. Bilateral striatal necrosis associated with a novel mutation in the mitochondrial ND6 gene. Ann Neurol. 2003;54(4):527-530. doi:10.1002/ana.10682

38. Malfatti E, Bugiani M, Invernizzi F, et al. Novel mutations of ND genes in complex I deficiency associated with mitochondrial encephalopathy. Brain. 2007;130(7):18941904. doi:10.1093/brain/awm114

39. Wray CD, Friederich MW, du Sart D, et al. A new mutation in MT-ND1 m.3928G >C p.V208L causes Leigh disease with infantile spasms. Mitochondrion. 2013;13(6):656-661. doi:10.1016/j.mito.2013.09.004

40. Tang S, Wang J, Zhang W, et al. Transition to next generation analysis of the whole mitochondrial genome: a summary of molecular defects. Hum Mutat. 2013;34(6):882-893. doi:10.1002/humu.22307

41. Chae JH, Lee JS, Kim KJ, Hwang YS, Hirano M. Biochemical and genetic analysis of Leigh syndrome patients in Korea. Brain Dev. 2008;30(6):387-390. doi:10.1016/j. braindev.2007.11.001

42. Lamperti C, Diodato D, Lamantea E, et al. MELAS-like encephalomyopathy caused by a new pathogenic mutation in the mitochondrial DNA encoded cytochrome c oxidase subunit I. Neuromuscul Disord. 2012;22(11):990-994. doi:10.1016/j.nmd.2012.06.003

43. Yoneda M, Tanno Y, Horai S, et al. A common mitochondrial DNA mutation in the tRNALys of patients with myoclonus epilepsy associated with ragged-red fibers. Biochem Int. 1990;21:789-796. 